\title{
Superinterpolation in highly oscillatory quadrature
}

\author{
Daan Huybrechs and Sheehan Olver
}

Report TW569, June 2010

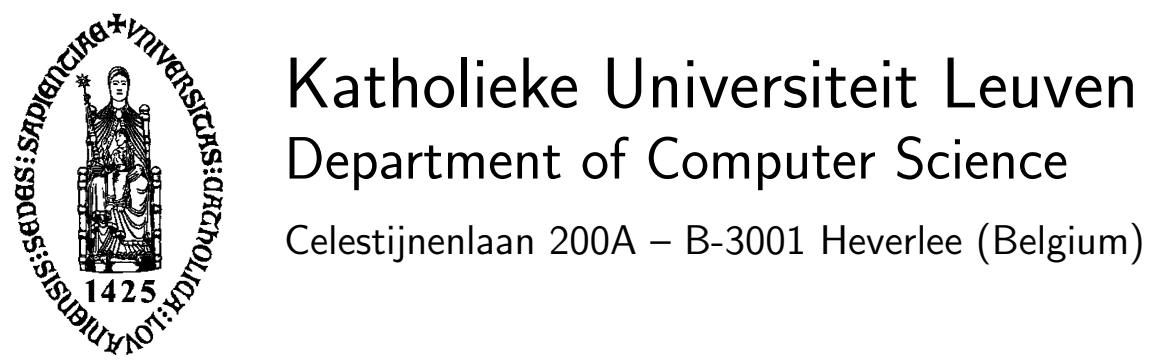




\title{
Superinterpolation in highly oscillatory quadrature
}

\author{
Daan Huybrechs and Sheehan Olver
}

Report TW569, June 2010

Department of Computer Science, K.U.Leuven

\begin{abstract}
Asymptotic expansions for oscillatory integrals typically depend on the values and derivatives of the integrand at a small number of critical points. We show that using values of the integrand at certain complex points close to the critical points can actually yield a higher asymptotic order approximation to the integral. This superinterpolation property has interesting ramifications for numerical methods based on exploiting asymptotic behaviour. The asymptotic convergence rates of Filon-type methods can be doubled at no additional cost. Numerical steepest descent methods already exhibit this high asymptotic order, but their analyticity requirements can be significantly relaxed. The method can be applied to general oscillators with stationary points as well, through a simple change of variables.
\end{abstract}

Keywords : oscillatory integral, steepest descent, Filon quadrature, numerical integration

AMS(MOS) Classification : Primary : 65D30 Secondary : 30E20, 41A60. 


\title{
Superinterpolation in highly oscillatory quadrature
}

\author{
Daan Huybrechs and Sheehan Olver
}

June 21, 2010

\begin{abstract}
Asymptotic expansions for oscillatory integrals typically depend on the values and derivatives of the integrand at a small number of critical points. We show that using values of the integrand at certain complex points close to the critical points can actually yield a higher asymptotic order approximation to the integral. This superinterpolation property has interesting ramifications for numerical methods based on exploiting asymptotic behaviour. The asymptotic convergence rates of Filon-type methods can be doubled at no additional cost. Numerical steepest descent methods already exhibit this high asymptotic order, but their analyticity requirements can be significantly relaxed. The method can be applied to general oscillators with stationary points as well, through a simple change of variables.
\end{abstract}

\section{Introduction}

We are interested in the numerical evaluation of highly oscillatory integrals of the form

$$
I[f]:=\int_{a}^{b} f(x) e^{i \omega g(x)} d x .
$$

Oscillatory integrals appear in several scientific disciplines and this model form has been widely studied in asymptotic analysis for large $\omega[20,2,25]$. Classical methods of numerical integration are costly, since they depend on sampling the integrand at a large number of points which must scale with $\omega$. For sufficiently smooth functions $f$ and $g$ however, the integral $I[f]$ typically has an asymptotic expansion in inverse powers of the frequency parameter $\omega$. Truncating this expansion after a few terms yields an approximation that improves with increasing $\omega$, at a fixed computational cost regardless of $\omega$. However, these expansions typically diverge when more terms are added and hence the numerical error is uncontrollable. Recent numerical methods take advantage of the asymptotic behaviour of the integral, without explicitly constructing the diverging expansion, and they can combine a fixed computational cost and high asymptotic order with numerical convergence. 
In this paper we consider Filon-type quadrature $[17,16]$ and steepest descent methods $[15,7]$. We refer the interested reader to [14] for a review of these and other methods.

The mentioned methods have high asymptotic order, by which we mean that the error of the method behaves as $\omega^{-\alpha}$ for $\omega \gg 1$. The steepest descent based method of [15] has the highest asymptotic order among all known methods. For the same number of evaluations of the integrand or its derivatives, the asymptotic order is almost twice that of the other methods. This doubling effect is related to the properties of Gaussian quadrature. The topic of this paper is the observation that the asymptotic order of the other methods can also be doubled by using the 'right' information of the integrand. These methods typically depend on using derivatives of the integrand at certain critical points. We will show that the asymptotic order improves simply by replacing evaluations of derivatives at critical points with evaluations at Gaussian quadrature points that are located in the complex plane and that can be obtained from a steepest descent analysis.

We make this explicit using a simple example. Consider the linear oscillator $g(x)=x$. In this case, the integral (1.1) depends asymptotically on the behaviour of the integrand near the endpoints $a$ and $b$. Filon-type methods are constructed by interpolating the function $f$ by a polynomial $p$ and then defining the approximation as the exact evaluation of the result,

$$
Q^{F}[f]=I[p] .
$$

High asymptotic order results from interpolating a number of derivatives of $f$ at $a$ and $b$ [17]. Interpolating $f$ and derivatives up to order $n-1$ at both endpoints yields an error that behaves in our example case as

$$
Q^{F}[f]-I[p]=\mathcal{O}\left(\omega^{-(n+1)}\right), \quad \omega \rightarrow \infty .
$$

The classical method of steepest descent is based on deforming the path of integration onto paths of steepest descent. These are paths in the complex plane originating at the critical points, along which $g(x)$ has constant real part and growing imaginary part. The integrand is then non-oscillatory and exponentially decaying. In the case $g(x)=x$, assuming $f$ is sufficiently analytic and has at most exponential growth in the complex plane and $\omega$ is sufficiently large, we can utilize Cauchy's theorem to obtain

$$
\begin{aligned}
\int_{a}^{b} f(x) e^{i \omega x} d x & =i \int_{0}^{\infty} f(a+i p) e^{-\omega p} d p-i \int_{0}^{\infty} f(b+i p) e^{-\omega p} d p \\
& =\frac{i}{\omega} \int_{0}^{\infty} f\left(a+i \frac{q}{\omega}\right) e^{-q} d q-\frac{i}{\omega} \int_{0}^{\infty} f\left(b+i \frac{p}{\omega}\right) e^{-q} d q .
\end{aligned}
$$

Following the approach of [15] we proceed by numerically evaluating the two resulting integrals with classical Gauss-Laguerre quadrature, i.e., using $e^{-q}$ 
as a weight function. It turns out that the typical high polynomial order of Gaussian quadrature translates into high asymptotic order of this approximation. Using $n$ points for both integrals, the error behaves as $\mathcal{O}\left(\omega^{-2 n-1}\right)$. This order is twice as high as the Filon-type method outlined above, while using the same number of function evaluations. Note that in the steepest descent method, we end up evaluating $f$ at the points

$$
\left\{a+i \frac{x_{n j}}{\omega}\right\}_{j=1}^{n} \cup\left\{b+i \frac{x_{n j}}{\omega}\right\}_{j=1}^{n},
$$

where $x_{n j}$ are the $n$ roots of the Laguerre polynomial of degree $n$.

The first observation we make is that the numerical steepest descent method can be reinterpreted as a Filon-type method with complex interpolation points. This can be easily seen as follows. From the properties of Gaussian quadrature, it is clear that the steepest descent approach is exact for polynomials up to degree $2 n-1$. Next, consider the polynomial $p$ that interpolates $f$ precisely at the points (1.3) and define the Filon-type method like before as the exact integral $I[p]$ of this polynomial. The interpolating polynomial has degree $2 n-1$ and therefore the method is also exact for polynomials up to degree $2 n-1$. It follows that the Filon-type method and the numerical steepest descent method in this example are exactly the same. Thus, the Filon-type method with complex interpolation points also enjoys the high asymptotic order $2 n+1$.

The second, and perhaps more surprising, observation we make is that this asymptotic order is maintained when adding interpolation points elsewhere. This has important consequences for both methods:

1. The asymptotic order of Filon-type methods can be doubled by replacing evaluations of derivatives at critical points by evaluations at certain points in the complex plane.

2. The numerical method of steepest descent can be made to converge, even in the presence of singularities of $f$ and $g$ in the complex plane, by adding points on $[a, b]$. It is sufficient that $f$ and $g$ are analytic in a small neighbourhood of $[a, b]$, instead of an infinitely large sector of the complex plane.

In this paper we intend to show that the superinterpolation property holds for general oscillatory integrals. We describe known superinterpolation points in $\S 2$. We prove the high asymptotic order of resulting complex Filontype methods in $\S 3$, starting with the model case $g(x)=x^{r}$. Next, we examine the combination of superinterpolation points with $m$ Chebyshev points on $[a, b]$ to obtain a numerically convergent scheme in $\S 4$. In $\S 5$ we analyse the behaviour of the error for the case $g(x)=x$ in the two limits $m \rightarrow \infty$ and $\omega \rightarrow \infty$. This includes the important result that the behaviour of the error as $m \rightarrow \infty$ decays as $\omega$ grows, and vice-versa; the behaviour as 
$\omega \rightarrow \infty$ decays as $m$ grows. We describe an approach for general oscillators $g(x)$ in $\S 6$, by constructing a map of the oscillator to $x^{r}$. Finally, we conclude the paper with some numerical experiments in $\S 7$.

\section{Asymptotic superinterpolation points}

Superinterpolation points correspond to the nodes of Gaussian quadrature rules in the complex plane, i.e., they are the roots of orthogonal polynomials with respect to a complex weight function.

Consider the functional

$$
L_{\Gamma, r}[f]:=\int_{\Gamma} f(z) e^{i z^{r}} d z,
$$

For certain choices of the integer $r$ and the integration contour $\Gamma$, an orthogonal polynomial sequence $p_{n}$ (OPS) exists where the polynomials are orthogonal with respect to this functional:

$$
L_{\Gamma, r}\left[p_{k} p_{l}\right]=\delta_{k l} .
$$

In the following, we take $\Gamma$ to be a path of steepest descent or a combination of steepest descent paths at the origin. There are $r$ such paths, corresponding to the $r$ roots of unity. They are straight lines through the origin at angles

$$
\alpha_{j}:=2 \pi i \frac{j}{r}+\frac{\pi i}{2 r}, \quad j=1, \ldots, r .
$$

The corresponding paths can be parameterized by

$$
h_{j}(t)=\alpha_{j} t, \quad 0 \leq t<\infty .
$$

The weight $e^{i x^{r}}$ becomes $e^{-t^{r}}$ along each of these paths.

We denote the roots of $p_{n}(x)$ by $x_{n, j}, j=1, \ldots, n$. The importance of these roots is summarized in the following lemma.

Lemma 2.1. [7, Lemma's 1,2] Consider a positive number $r$ and a quadrature rule with nodes $\mathbf{x}_{s}=\left(x_{1}, \ldots, x_{s}\right)$ and weights $\mathbf{w}_{s}=\left(w_{1}, \ldots, w_{s}\right)$ such that

$$
\int_{\Gamma} z^{m} e^{i z^{r}} d x=\sum_{k=1}^{s} w_{k} x_{k}^{n}, \quad m=0, \ldots, d-1,
$$

where $\Gamma$ is a steepest descent path at the origin, or a concatenation of two steepest descent paths at the origin. If $f$ is analytic at $z=0$ and if $\int_{\Gamma} f(z) e^{i \omega z^{r}} d z$ exists for $\omega \geq \omega_{0}$, then

$$
\int_{\Gamma} f(z) e^{i \omega z^{r}} d z-Q_{r}^{G}\left[f, \mathbf{x}_{s}, \mathbf{w}_{s}\right]=\mathcal{O}\left(\omega^{-\frac{d+1}{r}}\right),
$$


for

$$
Q_{r}^{G}\left[f, \mathbf{x}_{s}, \mathbf{w}_{s}\right]=\omega^{-1 / r} \sum_{k=1}^{s} w_{k} f\left(x_{k} \omega^{-1 / r}\right) .
$$

It is beneficial to use numerical quadrature to evaluate steepest descent integrals. The use of Gaussian quadrature maximizes the polynomial order $d$ of the quadrature rule and, by the lemma above, also the asymptotic order of the numerical approximation of steepest descent integrals.

We list the most interesting known combinations of $\Gamma$ and $r$.

1. The steepest descent path for $r=1$ leads to the case described in the introduction. The weight becomes $e^{-t}$ along $h_{0}(t)$ with $t \in[0, \infty)$. The orthogonal polynomials are rotations of the Laguerre polynomials and their roots correspond precisely to Gauss-Laguerre quadrature nodes, rotated onto the positive imaginary axis. These points are suitable to treat a regular endpoint of an oscillatory integral.

2. The case where $r=2$ and $\Gamma$ is the concatenation of $h_{0}(t)$ and $h_{1}(t)$, i.e., $\Gamma$ is an infinite straight line through the origin at an angle of $\pi / 4$, leads to rotated Hermite polynomials. The weight $e^{i x^{2}}$ becomes the classical Hermite weight function $e^{-t^{2}}$ along this line with $t \in(-\infty, \infty)$. The roots are suitable to treat a stationary point of order 1 in the interior of the integration interval.

3. Case 1 can be generalized to larger integer values of $r$. The path $h_{0}(t)$ is a halfline at an angle of $\pi /(2 r)$ and yields a so-called Freud-type weight function $e^{-t^{r}}$ with $t \in[0, \infty)$. The existence of the associated OPS is guaranteed by standard theory of orthogonal polynomials, because the weight is strictly positive along the contour. This case is suitable for stationary points of order $r-1$ that coincide with a left endpoint. A right endpoint is similar but uses the path $h_{\lfloor r / 2\rfloor}$.

4. Case 2 can be generalized to larger even values of $r$, say $r=2 q$. In this case, we can combine $h_{0}(t)$ and $h_{q}(t)$ into a single straight line through the origin at an angle of $\pi /(4 q)$. This leads to a generalization of Gauss-Hermite quadrature. The weight $e^{-t^{2 q}}$ with $t \in(-\infty, \infty)$ is again real and positive along $\Gamma$. This case is suitable for stationary points of odd order in the interior of the integration interval.

5. If $r=2 q+1$ is odd, then the combination of the two halflines $h_{0}(t)$ and $h_{q}(t)$ does not lead to a single straight line through the origin. In this case the functional can not be rewritten in the form of a single integral with a strictly positive weight function. It can be shown that the functional $L_{\Gamma, r}$ is indefinite [7]. However, the orthogonal polynomials do exist and their roots are suitable for degenerate stationary points of even order in the interior of the integration interval. 


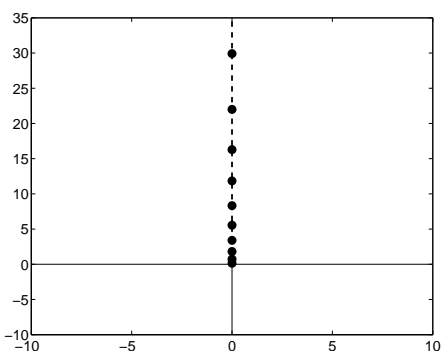

(a) Case 1: a regular endpoint

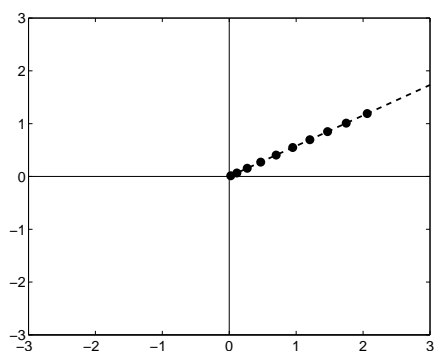

(c) Case 3: left endpoint and stationary point of order $2(r=$ 3)

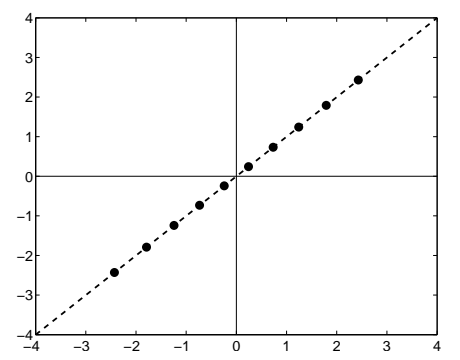

(b) Case 2: interior stationary point of order $1(r=2)$

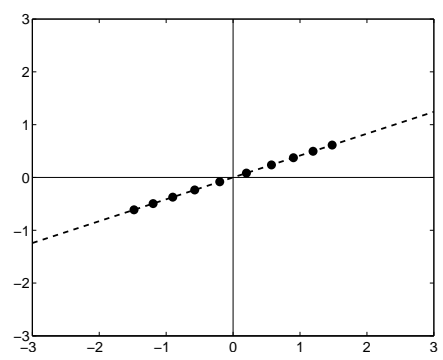

(d) Case 4: interior stationary point of order $3(r=4)$

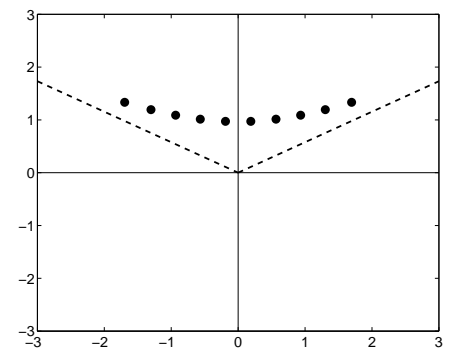

(e) Case 5: interior stationary point of order $2(r=3)$

Figure 1: Illustration of the roots of the polynomials orthogonal with respect to the functional $L_{\Gamma, r}$. The contour $\Gamma$ is shown in striped lines, the value of $r$ is indicated in the subcaption and $n=10$ points are shown in each figure.

The location of the points is shown in Figure 1. All points are located on a path of steepest descent, except in case 5 because in this case $L_{\Gamma, r}$ is not related to a positive definite functional on $\Gamma$. Instead, the points are located on a curve in a sector of the complex plane bounded by $h_{0}(t)$ and $h_{q}(t)$. For the case $r=3$, the curve and the asymptotic distribution of the points (for large degree of the polynomials) was determined explicitly in [8]. The existence of these polynomials for all $n$ has not been proved and an equivalent to the Golub-Welsch algorithm [13] has not been developed. 
This is a minor issue for the method we will develop, as we only require a small number of nodes to obtain the desired asymptotic order. The existence of the first few polynomials is easily verified by direct computation and the corresponding roots need be computed only once.

The use of Gauss-Laguerre quadrature along steepest descent paths for oscillatory integrals has been described several times in literature before, see $[24,5,3]$. An asymptotic analysis of this approach for oscillatory integrals of the form (1.1) is given in [15]. The quadrature points for the other cases are described in more detail in [7].

\section{Complex Filon-type methods}

The points described in $\S 2$ were previously used only to evaluate steepest descent integrals. Here, we describe and analyse a complex Filon-type method based on polynomial interpolation in these complex points and a number of additional points on the real line. We start with a formal description of the numerical steepest descent method.

\subsection{Numerical steepest descent method}

For simplicity of the presentation, we restrict our attention to an integral of the form

$$
I[f]:=\int_{-1}^{1} f(x) e^{i \omega x^{r}} d x .
$$

The oscillator $x^{r}$ has steepest descent paths in the directions given by (2.3). Two directions of interest are $\alpha_{0}$ and $\alpha_{\lfloor r / 2\rfloor}$, because the integral along the real line can be deformed onto steepest descent paths in these directions. Indeed, assuming $f$ is entire and has not more than exponential growth, we can use Cauchy's theorem to obtain, for all $\omega \geq \omega_{0}>0$,

$$
I[f]=\left(\int_{\Gamma_{-1}}+\int_{\Gamma_{0}}+\int_{\Gamma_{1}}\right) f(z) e^{i \omega z^{r}} d z .
$$

where the paths tend to infinity in the following way:

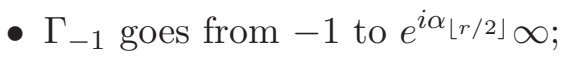

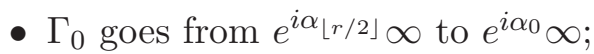

- $\Gamma_{1}$ goes from $e^{i \alpha_{0}} \infty$ to 1 .

These paths are clearly connected and the integrand decays exponentially towards infinity in these directions. Recall that $\Gamma_{0}$ may be taken to be a straight line through the origin for even values of $r$. 
A local substitution puts each line integral into the form (2.5) suitable for applying Gaussian quadrature,

$$
\begin{aligned}
u_{-1}(y) & =e^{2 \pi i\lfloor r / 2\rfloor / r} \sqrt[r]{(-1)^{r}+y}, \\
u_{0}(y) & =y \\
u_{1}(y) & =\sqrt[r]{1+y} .
\end{aligned}
$$

The integrals at the endpoints lead to the case of Laguerre-type polynomials (case 1 in $\S 2$ ). We denote the nodes and weights by $\mathbf{x}_{s}^{L}$ and $\mathbf{w}_{s}^{L}$. The integral along $\Gamma_{0}$ leads to the case of Hermite-type polynomials (cases 2,4,5 in $\S 2$ ) and we denote the nodes and weights by $\mathbf{x}_{r, s}^{H}$ and $\mathbf{w}_{r, s}^{H}$. Using $t$ points for the endpoint integrals and $s$ points for the stationary point contribution, the numerical steepest descent approximation is given by

$$
\begin{aligned}
Q_{s, t}^{S D}[f]= & e^{i \omega(-1)^{r}} Q_{1}^{G}\left[u_{-1}^{\prime} f \circ u_{-1}, \mathbf{x}_{t}^{L}, \mathbf{w}_{t}^{L}\right]+ \\
& Q_{r}^{G}\left[u_{0}^{\prime} f \circ u_{0}, \mathbf{x}_{r, s}^{H}, \mathbf{w}_{r, s}^{H}\right]+e^{i \omega} Q_{1}^{G}\left[u_{1}^{\prime} f \circ u_{1}, \mathbf{x}_{t}^{L}, \mathbf{w}_{t}^{L}\right] .
\end{aligned}
$$

It follows immediately from Lemma 2.1 that

$$
I[f]-Q_{s, t}^{S D}[f]=\mathcal{O}\left(\omega^{-(2 t+1)}\right)+\mathcal{O}\left(\omega^{-\frac{2 s+1}{r}}\right) .
$$

The errors can be matched by selecting

$$
t=\left\lceil\frac{2 s+1-r}{2 r}\right\rceil \text {. }
$$

With this choice, we have

$$
I[f]-Q_{s, t}^{S D}[f]=\mathcal{O}\left(\omega^{-\frac{2 s+1}{r}}\right) .
$$

The relative error scales as $\mathcal{O}\left(\omega^{-\frac{2 s}{r}}\right)$.

\subsection{Complex Filon-type methods}

In the steepest descent method (3.2) the function $f$ is evaluated at the following set of points, with $t$ related to $s$ as in (3.3),

$$
\mathbf{x}_{s}^{S I}=u_{-1}\left(\mathbf{x}_{t}^{L} \omega^{-1}\right) \cup u_{0}\left(\mathbf{x}_{r, s}^{H} \omega^{-1 / r}\right) \cup u_{1}\left(\mathbf{x}_{t}^{L} \omega^{-1}\right) .
$$

These are the superinterpolation points for this integral.

Lemma 3.1. Let $\left(\mathbf{x}_{s}, \mathbf{w}_{s}\right)$ be a quadrature rule of order d as in Lemma 2.1. Fix $\epsilon>0$ and let $\tilde{\Gamma}=\Gamma \cap B(\epsilon)$, where $B(\epsilon)$ is an open ball of size $\epsilon$. Suppose a function $p$ is analytic, bounded and with bounded derivatives as $\omega \rightarrow \infty$ in $B(\epsilon)$, and $p$ vanishes at the nodes $\mathbf{x}_{s} \omega^{-1 / r}$. Then

$$
\int_{\tilde{\Gamma}} p(z) e^{i \omega z^{r}} d z=\mathcal{O}\left(\omega^{-\frac{d+1}{r}}\right) .
$$


Proof. Since $p$ is analytic in $B(\epsilon)$ it has a Taylor series at $z=0$. We write $p$ as the sum of the first $d$ terms of its Taylor series and a remainder term,

$$
p(z)=p_{d}(z)+p_{r}(z) .
$$

Note that $p_{r}^{(j)}(0)=0, j=0, \ldots, d-1$, and therefore $p_{r}\left(x_{s, j} \omega^{-1 / r}\right)=$ $\mathcal{O}\left(\omega^{-d / r}\right)$. Since $p\left(x_{s, j} \omega^{-1 / r}\right)=0$, we also have $p_{d}\left(x_{s, j} \omega^{-1 / r}\right)=\mathcal{O}\left(\omega^{-d / r}\right)$.

We have, using $\mathcal{O}\left(\omega^{-\infty}\right)$ to denote superalgebraic decay,

$$
\begin{aligned}
\int_{\tilde{\Gamma}} p_{d}(z) e^{i \omega z^{r}} d z & =\int_{\Gamma} p_{d}(z) e^{i \omega z^{r}} d z+\mathcal{O}\left(\omega^{-\infty}\right) \\
& =Q_{r}^{G}\left[p_{d}, \mathbf{x}_{s}, \mathbf{w}_{s}\right]+\mathcal{O}\left(\omega^{-\infty}\right) \\
& =\mathcal{O}\left(\omega^{-\frac{d+1}{r}}\right)+\mathcal{O}\left(\omega^{-\infty}\right)=\mathcal{O}\left(\omega^{-\frac{d+1}{r}}\right) .
\end{aligned}
$$

Next, applying integration by parts $d+1$ times and using $p_{r}^{(j)}(0)=0$, $j=0, \ldots, d-1$, and boundedness of $p_{r}$ and its derivatives, we also have

$$
\int_{\tilde{\Gamma}} p_{r}(z) e^{i \omega z^{r}} d z=\mathcal{O}\left(\omega^{-\frac{d+1}{r}}\right) .
$$

This proves the result.

Theorem 3.2. Suppose a function p, bounded and with bounded derivatives for $\omega \rightarrow \infty$, interpolates $f$ at the superinterpolation points $\mathbf{x}_{s}^{S I}$. If $f$ and $p$ are analytic in an open simply connected domain $\Omega$ containing $[-1,1]$, then

$$
I[f]-I[p]=\mathcal{O}\left(\omega^{-\frac{2 s+1}{r}}\right) .
$$

Proof. Assume that $\omega$ is large enough so that $\mathbf{x}_{s}^{S I} \subset \Omega$. Fix $\epsilon>0$ and define $\Gamma_{x, \epsilon}:=\Gamma_{x} \cap B(x, \epsilon)$, where $B(x, \epsilon)$ is an open ball of radius $\epsilon$ centered at $x$. Assume that $\epsilon$ is sufficiently small, so that we may write

$$
I[f-p]=\left(\int_{\Gamma_{-1, \epsilon}}+\int_{\Gamma_{-1 \rightarrow 0}}+\int_{\Gamma_{0, \epsilon}}+\int_{\Gamma_{0 \rightarrow 1}}+\int_{\Gamma_{1, \epsilon}}\right)(f(z)-p(z)) e^{i \omega z^{r}} d z .
$$

where $\Gamma_{-1 \rightarrow 0}$ and $\Gamma_{0 \rightarrow 1}$ are straight lines in $\Omega$ connecting the endpoints of $\Gamma_{ \pm 1, \epsilon}$ and $\Gamma_{0, \epsilon}$. The second and fourth integrals are exponentially small, hence do not contribute to the asymptotics. The theorem then follows from applying Lemma 3.1 to the remaining integrals.

For any given set of points $\mathbf{x}^{F}$, not depending on $\omega$, we can construct the polynomial interpolating $f$ in the points

$$
\mathbf{x}^{F} \cup \mathbf{x}_{s}^{S I} .
$$


As $\omega \rightarrow \infty$, the complex interpolation points converge to \pm 1 and zero, hence the polynomial converges to a Hermite interpolation polynomial independent of $\omega$. It follows that we can apply Theorem 3.2. We approximate $I[f]$ by

$$
Q^{C}[f]=I[p]
$$

and may conclude that

$$
I[f]-Q^{C}[f]=\mathcal{O}\left(\omega^{-\frac{2 s+1}{r}}\right) .
$$

\section{Chebyshev interpolation}

The error estimate (3.5) is very encouraging, but only asymptotic. Historically, the analysis of Filon quadrature has focused mostly on the interpolation error. Indeed, a crude but non-asymptotic error bound can be obtained using simply that $\left|e^{i \omega g(x)}\right|=1$,

$$
|I[f]-I[p]| \leq|b-a| \max _{x \in[a, b]}|f(x)-p(x)| .
$$

It will be clear from the numerical experiments that the estimates (3.5) and (4.1) do not completely describe the subtleties of the convergence characteristics of our scheme. For the simplest oscillator $g(x)=x$, we provide a partial answer in $\S 5$ by analysing the asymptotics as the number of interpolation points increase and as $\omega$ increases, separately. For a more detailed analysis of Filon-type quadrature, including estimates in different regimes (though none of which demonstrate the high asymptotic order we achieve in this approach), we refer the reader to [19].

Still, it is evident from (4.1) and (3.5) that complex Filon quadrature enables a convergent scheme with high asymptotic order by controlling the interpolation error. For this reason we combine the superinterpolation points in the complex plane with the Chebyshev nodes on $[-1,1]$,

$$
\mathbf{x}_{m}^{T}=\left\{\cos \frac{(2 m-1) \pi}{2 m}, \cos \frac{(2 m-3) \pi}{2 m}, \ldots, \cos \frac{\pi}{2 m}\right\} .
$$

We assume for simplicity as before that $[a, b]=[-1,1]$ and $g(x)=x^{r}$.

We are thus left with the problem of computing $I[p]$. The simplest approach is arguably to express the interpolant as

$$
p(x)=\sum c_{k} x^{k}
$$

so that

$$
I[p]=\sum c_{k} I\left[x^{k}\right]
$$


and the latter terms $I\left[x^{k}\right]$ can be explicitly computed for oscillators of the form $g(x)=x^{r}$ in terms of the (incomplete) Gamma function and hypergeometric functions. Unfortunately, this is not numerically stable as $m$ or $s$ go to infinity, due to significant cancellation [11]. The approach is feasible only with high-precision calculations.

To obtain a numerically stable algorithm, we want to express $p(x)$ as its Chebyshev expansion,

$$
p(x)=\sum c_{k} T_{k}(x) .
$$

Then we can use the following fast algorithm.

Algorithm 4.1 (Complex Filon-type method).

Given $f$, integer $m$ and $n$ additional points $\mathbf{x}=\left(x_{1}, \ldots, x_{n}\right)^{T}$.

Step 1: Define $\mathbf{T}_{m}$ as $\left(T_{0}, \ldots, T_{m}\right)$.

Step 2: Interpolate $f$ by a polynomial $p_{1}=\mathbf{T}_{m-1} \mathbf{p}_{1}$ at $m$ Chebyshev points, using the fast cosine transform.

Step 3: Interpolate $q=\left(f-p_{1}\right) / T_{m}$ at the points $\mathbf{x}$ by a polynomial $p_{2}$ using the barycentric interpolation formula:

$$
p_{2}(x)=\frac{\sum_{k=1}^{n} \frac{w_{k}}{x-x_{k}} q\left(x_{k}\right)}{\sum_{k=1}^{n} \frac{w_{k}}{x-x_{k}}} \quad \text { for } \quad w_{k}=\frac{1}{\prod_{j=1, j \neq k}^{n}\left(x_{k}-x_{j}\right)} .
$$

Step 4: Expand $p_{2}$ into its Chebyshev series $p_{2}=\mathbf{T}_{n-1} \mathbf{p}_{2}$, again using the fast cosine transform.

Step 5: Using the identity

$$
2 T_{r} T_{q}=T_{r+q}+T_{|r-q|},
$$

write

$$
p_{3}=T_{m} p_{2}=\frac{1}{2} \sum p_{2, k}\left[T_{m+k}+T_{|m-k|}\right]=\mathbf{T}_{n+m-1} \mathbf{p}_{3} .
$$

Step 6: Padding $\mathbf{p}_{1}$ at the end with zeros, the polynomial which interpolates at $m$ Chebyshev points and the points $\mathbf{x}$ is then

$$
p_{4}=p_{1}+p_{3}=\mathbf{T}_{n+m-1}\left[\mathbf{p}_{1}+\mathbf{p}_{3}\right]=T_{n+m-1} \mathbf{p}_{4} .
$$

Thus we define

$$
Q^{C}[f]=I\left[p_{4}\right]=\mu_{n+m-1} \mathbf{p}_{4} \quad \text { for } \quad \mu_{p}=\left(\mu_{1}, \ldots, \mu_{p}\right),
$$

where

$$
\mu_{k}=\int_{-1}^{1} T_{k}(x) e^{i \omega x^{r}} d x
$$


This leaves the problem of evaluating the modified moments

$$
\mu_{k}=I\left[T_{k}\right] .
$$

This problem has received some study in the case $r=1$, i.e., in the absence of stationary points. A recurrence relation can be found using the properties of Chebyshev polynomials (see [9] and references therein). However, this becomes unstable for $k>\omega$. Moments for larger $k$ can be computed in a stable manner by solving a tridiagonal system of equations [9]. The case of moments in the presence of stationary points is largely unexplored.

Alternatively, since the function $f$ is replaced by a polynomial, we may simply use the original numerical steepest descent method. There are no issues in the complex plane, since polynomials are entire functions. This approach is simple and succesful for small to moderate degrees of the interpolant, but in this case too instabilities may arise for larger degrees. The instability is due to the rapid growth of polynomials of large degree in the complex plane, which initially swamps the exponential decay of the oscillator.

In order to generate the numerical examples in this paper, we have used an accurate general-purpose numerical integration package Cubpack [4]. The stable computation of the modified moments (4.2) is a topic of further investigation.

\section{$5 \quad$ Error analysis}

We restrict ourselves to the case $g(x)=x$, using $m$ Chebyshev points and $s$ points near both endpoints. A general expression for the error of a quadrature rule was derived in [10], see also [6, §4.6]. This expression in our setting becomes:

$$
R_{m, s}=\frac{1}{2 \pi i} \int_{\Gamma} \frac{f(z)}{P_{m, s}(z)} \int_{-1}^{1} \frac{P_{m, s}(x)}{z-x} e^{i \omega x} d x d z .
$$

Here, $P_{m, s}$ is a polynomial that vanishes at all the quadrature points,

$$
P_{m, s}(x)=L_{s}(-i \omega-i \omega x) T_{m}(x) L_{s}(i \omega-i \omega x),
$$

with $T_{m}$ and $L_{s}$ the Chebyshev and Laguerre polynomials, and $\Gamma$ is a contour that contains $[-1,1]$ in its interior.

The error expression depends on the variables $m, s$ and $\omega$. We intend to apply the scheme only with $s$ small, but we are interested in its behaviour for large $m$ and $\omega$. Any analysis of the error in the presence of two variables that may grow large independently of each other rapidly leads to complicated uniform asymptotics. For this reason, we study the asymptotic behaviour for large $m$ and large $\omega$ separately, but in both cases we also investigate the dependence of the leading order term on the other parameter. 


\subsection{Large $m$ asymptotics explicit in $\omega$}

We start with an analysis of the behaviour of the Cauchy transform in (5.1),

$$
\begin{gathered}
\int_{-1}^{1} \frac{P_{m, s}(x)}{z-x} e^{i \omega x} d x=\int_{0}^{\pi} \frac{e^{i \omega \cos \theta} L_{s}(-i \omega-i \omega \cos \theta) L_{s}(i \omega-i \omega \cos \theta)}{z-\cos \theta} \\
\cos m \theta \sin \theta d \theta .
\end{gathered}
$$

The asymptotic behaviour of integrals of this form can be inferred via integration by parts. For a given smooth function $f$, we have from repeated integration by parts

$$
\begin{aligned}
\int_{0}^{\pi} & f(\theta) \sin \theta \cos m \theta d \theta=-\frac{1}{m} \int_{0}^{\pi}(f(\theta) \sin \theta)^{\prime} \sin m \theta d \theta \\
& =\left.\frac{1}{m^{2}} f(\theta) \cos \theta \cos m \theta\right|_{0} ^{\pi}-\frac{1}{m^{2}} \int_{0}^{\pi}\left(f^{\prime}(\theta) \sin \theta+f(\theta) \cos \theta\right)^{\prime} \cos m \theta d \theta \\
& =\frac{1}{m^{2}}\left[(-1)^{m+1} f(\pi)-f(0)\right]+\mathcal{O}\left(\frac{1}{m^{3}}\right) .
\end{aligned}
$$

This means that

$$
\int_{-1}^{1} \frac{P_{m, s}(x)}{z-x} e^{i \omega x} d x \sim \frac{1}{m^{2}}\left[(-1)^{m+1} \frac{e^{-i \omega} L_{s}(2 i \omega)}{z+1}-\frac{e^{i \omega} L_{s}(-2 i \omega)}{z-1}\right] .
$$

Note that we have applied the property $L_{s}(0)=1$.

We proceed with the analysis of the error term (5.1). An explicit expression for the Chebyshev polynomials is $[18, \S 1]$

$$
T_{m}(z)=\frac{1}{2}\left[\left(z+\sqrt{z^{2}-1}\right)^{m}+\left(z-\sqrt{z^{2}-1}\right)^{m}\right] .
$$

We consider a contour $\Gamma$ that is an ellipse with foci at \pm 1 . For $z$ on such an ellipse, the quantity $\rho=z+\sqrt{z^{2}-1}$ is a constant and we have the bound

$$
\frac{1}{2}\left(\rho^{m}-\rho^{-m}\right) \leq\left|T_{m}(z)\right| \leq \frac{1}{2}\left(\rho^{m}+\rho^{-m}\right) .
$$

We may conclude that $T_{m}(z)^{-1} \sim 2 \rho^{-m}$ and combined with (5.3) we find

$$
\begin{aligned}
\left|R_{m, s}\right| & \sim \frac{1}{2 \pi i} m^{-2} 2 \rho^{-m} \\
& \times\left|\int_{\Gamma} \frac{f(z) T_{m}(z)}{P_{m, s}(z)}\left[(-1)^{m+1} \frac{e^{-i \omega} L_{s}(2 i \omega)}{z+1}-\frac{e^{i \omega} L_{s}(-2 i \omega)}{z-1}\right] d z\right| \\
\leq & \frac{1}{\pi i} m^{-2} \rho^{-m} \int_{\Gamma} \frac{|f(z)|}{\left|L_{s}(-i \omega(z+1)) L_{s}(-i \omega(z-1))\right|} \\
& \times\left[\frac{\left|L_{s}(2 i \omega)\right|}{|z+1|}+\frac{\left|L_{s}(-2 i \omega)\right|}{|z-1|}\right] d z \\
= & C(\omega) m^{-2} \rho^{-m} .
\end{aligned}
$$


This shows that the leading order behaviour in $m$ is $m^{-2} \rho^{-m}$, where $\rho$ is as large as we may take it depending on the domain of analyticity of $f$. In order to obtain the dependence of the constant $C(\omega)$ on $\omega$, it suffices to note that each Laguerre polynomial in this expression behaves as $\omega^{s}$. Since

$$
L_{s}(x) \sim \frac{(-1)^{s}}{s !} x^{s}, \quad|x| \rightarrow \infty,
$$

we have for $\omega \gg 1$

$$
L_{s}(i \omega(z \pm 1)) \sim \frac{(-i)^{s}}{s !}(z \pm 1)^{s} \omega^{s}
$$

and

$$
L_{s}( \pm 2 i \omega) \sim \frac{(-2 i)^{s}}{s !} \omega^{s}
$$

It follows that the error has the following bound:

$$
\left|R_{m, s}\right| \leq D_{s}[f] \omega^{-s} m^{-2} \rho^{-m}
$$

for some constant $D_{s}[f]$ dependent only on $f$ and $s$. This shows that the fast convergence rate of Chebyshev expansions is combined with high asymptotic order in $\omega$, but the superinterpolation rate of $\omega^{-2 s-1}$ is not completely maintained in this regime.

\subsection{Large $\omega$ asymptotics explicit in $m$}

First, we show that $P_{m, s}(z)$ behaves as $\omega^{2 s}$. From (5.4) we have for $\omega \gg 1$,

$$
\begin{aligned}
P_{m, s}(x) & \sim \frac{(-1)^{s}}{s !}(-i \omega(x+1))^{s} T_{m}(x) \frac{(-1)^{s}}{s !}(-i \omega(x-1))^{s} \\
& =\frac{(-1)^{s}}{(s !)^{2}}\left(x^{2}-1\right)^{s} T_{m}(x) \omega^{2 s} .
\end{aligned}
$$

Next we consider the Cauchy transform in (5.1). Since $z$ is bounded away from $[-1,1]$, we have for $\omega \gg 1$ that

$$
\begin{aligned}
\int_{-1}^{1} \frac{P_{m, s}(x)}{z-x} e^{i \omega x} d x= & \left(\int_{\Gamma_{1}}+\int_{\Gamma_{2}}\right) \frac{P_{m, s}(x)}{z-x} e^{i \omega x} d x \\
& + \begin{cases}2 \pi i P_{m, s}(z) e^{i \omega z} & -1<\Re z<1 \text { and } \Im z>0 \\
0 & \text { otherwise }\end{cases}
\end{aligned}
$$

where $\Gamma_{1}$ and $\Gamma_{2}$ are the steepest descent paths at -1 and 1 respectively. We do not define this deformation for $\Re z= \pm 1$ as a single point does not contribute to the integral over $\Gamma$ (though one could do so by using principal value integrals). The last term is exponentially small as $\omega \rightarrow \infty$, hence will 
not contribute to the asymptotic expansion. We now concentrate on the integral along $\Gamma_{1}$. Parameterizing by $x=-1+i t / \omega$, we find

$$
\int_{\Gamma_{1}} \frac{P_{m, s}(x)}{z-x} e^{i \omega x} d x=\frac{e^{-i \omega}}{\omega} \int_{0}^{\infty} \frac{L_{s}(t) T_{m}(-1+i t / \omega) L_{s}(2 i \omega+t)}{z-(-1+i t / \omega)} e^{-t} d t .
$$

We further expand

$$
\begin{aligned}
\frac{1}{z-(-1+i t / \omega)} & =\frac{1}{z+1} \frac{1}{1-\frac{i t}{\omega(z+1)}} \\
& =\frac{1}{z+1}\left(\sum_{k=0}^{s}(-i(z+1))^{-k}(t / \omega)^{k}+O\left(\omega^{-s-1}\right)\right) .
\end{aligned}
$$

For certain constants $c_{m, k}$ and $l_{k}$ we have

$$
\begin{aligned}
T_{m}(-1+i t / \omega) & L_{s}(2 i \omega+t) \\
& =\omega^{s} \sum_{k=0}^{m} c_{m, k}(t / \omega)^{k} \sum_{k=0}^{s}\left[l_{k}(t / \omega)^{k}+t^{k} O\left(\omega^{-k-1}\right)\right] \\
& =\omega^{s} \sum_{k=0}^{m+s} r_{m, k}(t / \omega)^{k}+\sum_{k=0}^{m+s} t^{k} O\left(\omega^{s-k-1}\right)
\end{aligned}
$$

where $r_{m, k}=\sum_{j=0}^{k} c_{m, j} l_{j-k}$. The second sum consist of higher order terms which will not contribute to the leading term asymptotics, hence we can drop them. Thus we get (using the orthogonality of $L_{s}(t)$ with lower degree polynomials)

$$
\begin{aligned}
(5.5) & \sim \frac{e^{-i \omega} \omega^{s-1}}{z+1} \int_{0}^{\infty} \sum_{k=0}^{m+s} r_{m, k}(t / \omega)^{k} \sum_{k=0}^{s}(-i(z+1))^{-k}(t / \omega)^{k} L_{s}(t) e^{-t} d t \\
& \sim \frac{e^{-i \omega} \omega^{s-1}}{z+1} \int_{0}^{\infty}(t / \omega)^{s} \sum_{k=0}^{s} r_{m, k}\left((-i(z+1))^{s-k} L_{s}(t) e^{-t} d t\right. \\
& =\frac{e^{-i \omega}}{\omega}(-i)^{s}(z+1)^{s-1} \int_{0}^{\infty} t^{s} L_{s}(t) e^{-t} d t \sum_{k=0}^{s} r_{m, k}(-i(z+1))^{-k} \\
& =\frac{e^{-i \omega}}{\omega} i^{s}(z+1)^{s-1} s ! \sum_{k=0}^{s} r_{m, k}(-i(z+1))^{-k} .
\end{aligned}
$$

The last integral is evaluated by writing $t^{s}=(-1)^{s} s ! L_{s}(t)+O\left(t^{s-1}\right)$. 
Thus we obtain the asymptotic expression (as $\omega \rightarrow \infty)$

$$
\begin{aligned}
& \int_{\Gamma} \frac{f(z)}{P_{m, s}(z)} \int_{\Gamma_{1}} \frac{P_{m, s}(x)}{z-x} e^{i \omega x} d x d z \\
& \sim \int_{\Gamma} \frac{f(z)}{P_{m, s}(z)} \frac{e^{-i \omega}}{\omega} i^{s}(z+1)^{s-1} s ! \sum_{k=0}^{s} r_{m, k}(-i(z+1))^{-k} d z \\
& \sim(-1)^{s} \frac{e^{-i \omega}}{\omega^{2 s+1}} i^{s}(s !)^{3} \sum_{k=0}^{s} r_{m, k} i^{k} \\
& \quad \times \int_{\Gamma} \frac{f(z)}{\left(z^{2}-1\right)^{s} T_{m}(z)}(z+1)^{s-k-1} d z
\end{aligned}
$$

We wish to show that this improves as $m$ increases. $r_{m, k}$ for $k=0, \ldots, s$ depend on $c_{m, k}$ also for $k=0, \ldots, s$. So the rate of growth is proportional to the worst such $c_{m, k}$. Note that that the Taylor series of $T_{m}(x)$ around -1 is

$$
T_{m}(x)=(-1)^{m} m \sum_{k=0}^{m}\left(\begin{array}{c}
m+k \\
m-k
\end{array}\right) \frac{(-2)^{k}}{k+m}(x+1)^{k} .
$$

This expression can be proved by using 18.7.3 (an expression for Chebyshev polynomials as Jacobi polynomials), 18.5.7 (the Taylor series of Jacobi polynomials at $x=+1$ and 5.5.5 (an expression of $\Gamma(n+1 / 2)$ in terms of $\Gamma(2 n)$ and $\Gamma(n))$ in [21]; along with the fact that $T_{m}(x)=(-1)^{m} T_{m}(-x)$.

Now from Stirlings formula we have

$$
\begin{aligned}
\left(\begin{array}{c}
m+k \\
m-k
\end{array}\right) & =\frac{(m+k) !}{(m-k) !(2 k) !} \sim \frac{\sqrt{m+k}\left(\frac{m+k}{e}\right)^{m+k}}{\sqrt{m-k}\left(\frac{m-k}{e}\right)^{m-k}(2 k) !} \\
& \sim \frac{\left(\frac{m+k}{m-k}\right)^{m}\left(m^{2}-k^{2}\right)^{k}}{(2 k) ! e^{2 k}} \sim \frac{\left(m^{2}-k^{2}\right)^{k}}{(2 k) !} .
\end{aligned}
$$

We thus have

$$
c_{m, k} \sim \frac{\left(m^{2}-k^{2}\right)^{k}}{(2 k) !} \frac{(-2 i)^{k}}{k+m} .
$$

Since $k$ ranges over $0, \ldots, s$, the largest growth is for $c_{m, s} \sim C_{s} m^{2 s-1}$, and this only contributes to $r_{m, s}$, hence we obtain (now for $m \rightarrow \infty$, and using that $\left.l_{0}=1\right)$ :

$$
(5.6) \sim D_{1, s}[f] \frac{e^{-i \omega}}{\omega^{2 s+1}} m^{2 s-1} \rho^{-m} .
$$

Similarly, the equivalent expression along $\Gamma_{2}$ behaves like

$$
D_{2, s}[f] \frac{e^{i \omega}}{\omega^{2 s+1}} m^{2 s-1} \rho^{-m} .
$$




\section{General oscillators}

Up until now, we have restricted our attention to oscillators of the form $x^{r}$. In this section, we will see that integrals with general oscillators can be rewritten in that form, and thus the results of the previous sections automatically apply. Consider the oscillatory integral

$$
I[f]:=\int_{a}^{b} f(x) e^{i \omega g(x)} d x
$$

where $a<0<b, f$ and $g$ are analytic in a neighbourhood of $(a, b), g(x) \sim x^{r}$ as $x \rightarrow 0$ and $g(x), g^{\prime}(x) \neq 0$ away from the stationary point at $x=0$. If instead $g$ has a single stationary point at $a<\xi<b$ of the form $g(\xi)=$ $g(\xi)+\frac{g^{(r)}(\xi)}{r !}(x-\xi)^{r}$ with $g(x) \neq g(\xi)$ away from $\xi$, we can put the integral into the desired form by writing

$$
\left.\int_{a}^{b} f(x) e^{i \omega g(x)} d x=e^{i \omega g(\xi)} \int_{a-\xi}^{b-\xi} f(x+\xi) e^{i\left(\omega \frac{g^{(r)}(\xi)}{r !}\right)\left[\frac{r !}{g^{(r)}(\xi)}(g(x+\xi)-g(\xi))\right.}\right] d x .
$$

If the integral contains multiple (but finitely many) stationary points or points where $g(x)=g(\xi)$, we can subdivide the interval into multiple integrals to impose the necessary conditions.

If the integral is free of stationary points (i.e., $r=1$ ), a simple transformation has been used effectively to map the integral to the canonical situation [11]. Define $u=g(x)$, thence

$$
I[f]=\int_{g(a)}^{g(b)} \frac{f\left(g^{-1}(u)\right)}{g^{\prime}\left(g^{-1}(u)\right)} e^{i \omega u} d u
$$

Since $g$ is monotonic, it is invertible (and its inverse is easily computable using e.g. Newton's method). Since $g^{\prime}$ does not vanish, the denominator in the integrand does not blow up.

For higher order $r$, the above approach does not work since $g$ is not necessarily uniquely invertible, and $g^{\prime}\left(g^{-1}(u)\right)$ vanishes, introducing a singularity. However, consider the following map:

$$
\sqrt[r]{g}(x):=x\left(\frac{g(x)}{x^{r}}\right)^{\frac{1}{r}}
$$

The notation is justified since $(\sqrt[r]{g})^{r}=g$, and we will see that it is an analytic continuation of $\sqrt[r]{g(x)}$ from $x$ positive to $x$ negative. This map was proposed in this form for the use in moment-free Filon-type methods and a differential GMRES method $[22,23]$. The way we will use the map is based on an earlier construction in [26], which split the interval and used the non-analytically continued map $\sqrt[r]{g(x)}$. 
Note that $\frac{g(x)}{x^{r}}=1+O(x)$. Since $g(x)$ does not vanish away from zero (and the reason we needed to make that assumption), we obtain $\frac{g(x)}{x^{r}}>$ 0 , and therefore $\sqrt[r]{g}$ is analytic in a neighbourhood of the unit interval. Furthermore,

$$
(\sqrt[r]{g})^{\prime}=\frac{g^{\prime}}{g} \sqrt[r]{g}
$$

cannot vanish anywhere except at zero, and at zero

$$
\frac{g^{\prime}}{g} \sqrt[r]{g} \sim r>0
$$

Thus $(\sqrt[r]{g})^{\prime}>0$ and $\sqrt[r]{g}$ is monotonic, and hence invertible.

We can now do the transformation $u=\sqrt[r]{g}(x)$, so that

$$
\begin{aligned}
I[f] & =\int_{\sqrt[r]{g}(a)}^{\sqrt[r]{g}(b)} \frac{f\left(\sqrt[r]{g}^{-1}(u)\right)}{\sqrt[r]{g^{\prime}}\left(\sqrt[r]{g}^{-1}(u)\right)} e^{i \omega u^{r}} d u \\
& =\int_{\sqrt[r]{g}(a)}^{\sqrt[r]{g}(b)} f\left(\sqrt[r]{g}^{-1}(u)\right) \frac{g\left(\sqrt[r]{g}^{-1}(u)\right)}{u g^{\prime}\left(\sqrt[r]{g}^{-1}(u)\right)} e^{i \omega u^{r}} d u .
\end{aligned}
$$

Note that $f\left(\sqrt[r]{g}^{-1}(u)\right) \sqrt[r]{g}^{\prime}\left(\sqrt[r]{g}^{-1}(u)\right)$ is analytic in a neighbourhood of $(\sqrt[r]{g}(a), \sqrt[r]{g}(b))$, since $\sqrt[r]{g}$ is analytic in a neighbourhood of $(a, b)$ and the inverse of a monotonic, analytic function is also monotonic and analytic in a sufficiently small neighbourhood. We have thus reduced the general oscillator to the form previously considered.

\section{Numerical results}

We illustrate the convergence characteristics of complex Filon-type methods with a number of experiments.

Consider first the integral without stationary points,

$$
I_{1}:=\int_{-1}^{1}(\cos x+\sin x) e^{i \omega x} d x .
$$

Fig. 2 illustrates numerical convergence both for increasing frequency $\omega$ and for increasing number of Chebyshev points $m$. In the left figures, convergence is plotted as a function of $\omega$. The errors decay as $\mathcal{O}\left(\omega^{-3}\right), \mathcal{O}\left(\omega^{-5}\right)$ and $\mathcal{O}\left(\omega^{-7}\right)$ respectively in Figures 2(a), (c) and (e). For larger $m$, the errors are small and they still decay with $\omega$. There appears to be a kink phenomenon in the three figures, most visible in Fig. 2(e). The asymptotic convergence rates match the smaller slopes at larger $\omega$. We currently have no explanation for the initial convergence that appears to be faster. In the right figures, convergence is shown as a function of $m$. Exponential convergence is seen 


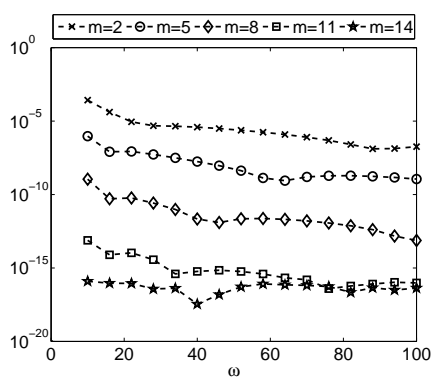

(a) $s=1$

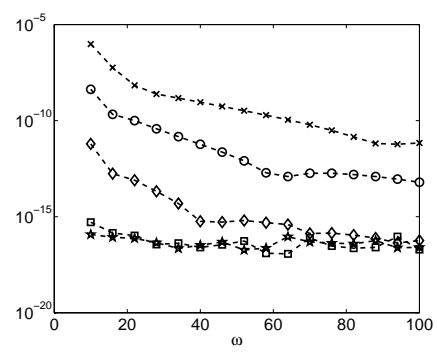

(c) $s=2$

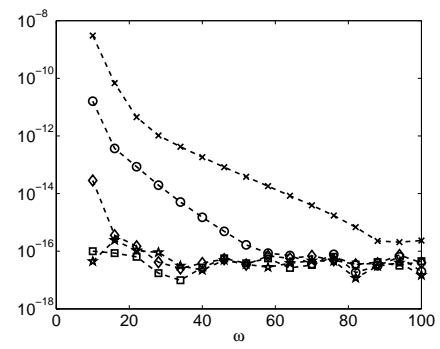

(e) $s=3$

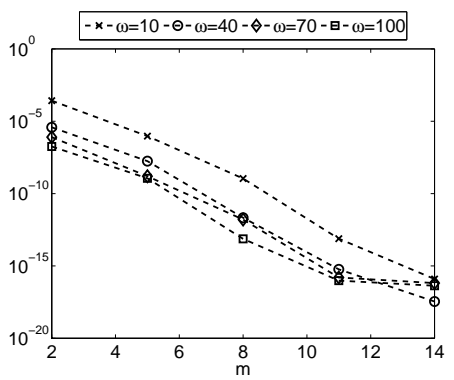

(b) $s=1$

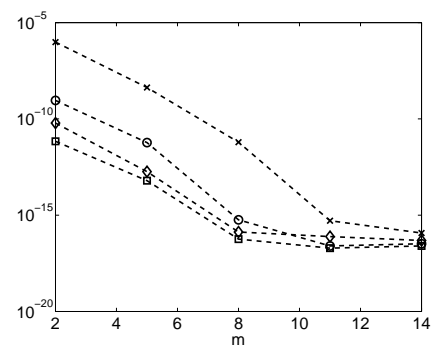

(d) $s=2$

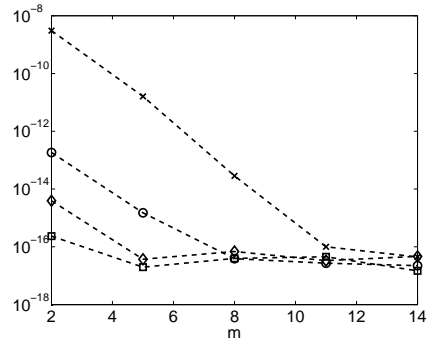

(f) $s=3$

Figure 2: Convergence of the superinterpolation scheme with Chebyshev points for the integral $\int_{-1}^{1}(\cos x+\sin x) e^{i \omega x} d x$ as a function of increasing $\omega$ (left figures) or as a function of increasing number of Chebyshev points $m$ (right figures).

in the three cases, that levels off only when machine precision is reached. Errors are consistently smaller for larger values of $\omega$.

We compare the results to Filon-type quadrature using interpolation of derivatives at the endpoints in Fig. 3. We have used function values and derivatives up to order 2 at the endpoints. This should be compared to using 3 superinterpolation points near both endpoints, as in Fig. 2(e)-(f). The errors in the superinterpolation scheme are smaller by several orders of magnitude, while the computational cost is the same since the degree of the interpolant is the same and an equal number of moments are used in both 


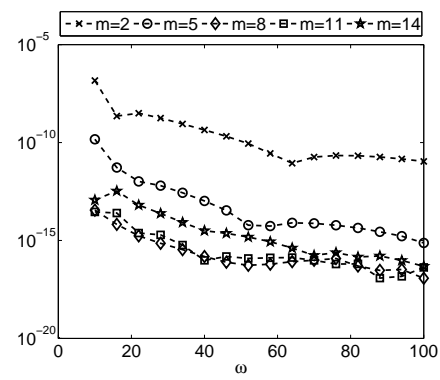

(a)

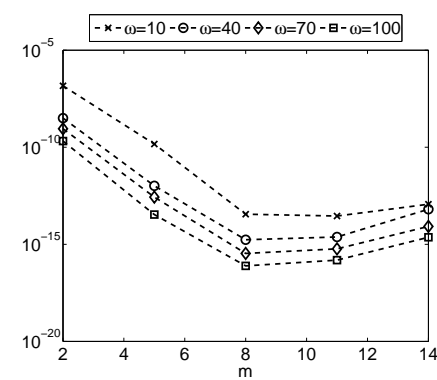

(b)

Figure 3: Similar setup as in Fig. 2, but with Filon-type quadrature using derivatives at the endpoints. Results are shown using Hermite interpolation of derivatives up to order 2 at the endpoints. This should be compared with Fig. 2(e)-(f), where 3 superinterpolation are used at both endpoints instead.

methods.

Next, consider the integral with a stationary point of order 2 ,

$$
I_{2}:=\int_{-1}^{1} \frac{1}{1+5 x^{2}} e^{i \omega x^{3}} d x .
$$

In addition, the integrand has a pole in the complex plane at $z=i / \sqrt{5}$. Results are shown in Fig. 4 both for increasing $\omega$ and for increasing $m$. Due to the pole close to the real axis, a larger number of Chebyshev points is needed in order to reach machine precision. However, the exponential convergence as a function of $m$ is clear. The asymptotic convergence rates in this example are again $\mathcal{O}\left(\omega^{-3}\right), \mathcal{O}\left(\omega^{-5}\right)$ and $\mathcal{O}\left(\omega^{-7}\right)$. These rates are exhibited in the left figures for small $m$. For larger $m$, the asymptotic regime is not fully reached within the range of $\omega$ shown in the figures. However, errors are consistently smaller for larger $m$. Note that the numerical method of steepest descent as proposed in [15] would not converge to the correct value of the integral unless the residue at $i / \sqrt{5}$ was taken into account explicitly. This issue is avoided with the currently proposed method: analyticity of the integrand in a neighbourhood of the endpoints is sufficient since interpolation at Chebyshev points distributed on the real axis ensures numerical convergence. We may conclude that the vicinity of the pole affects the convergence rate, but not convergence itself.

\section{References}

[1] J.-P. Berrut and L. Trefethen. Barycentric lagrange interpolation. SIAM Review, 46:501-517, 2004. 


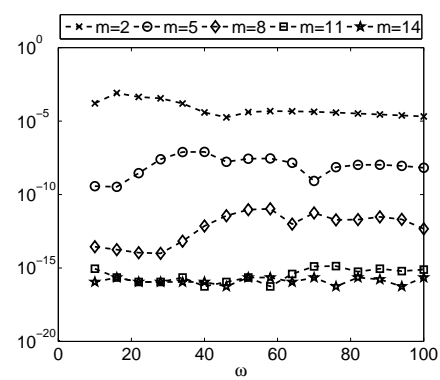

(a) $s=4$

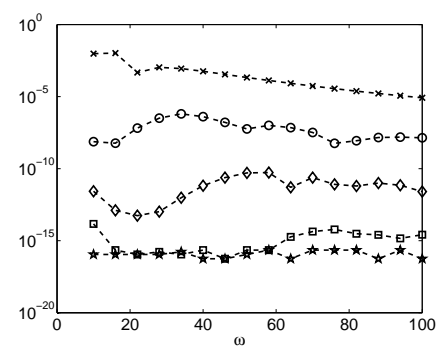

(c) $s=7$

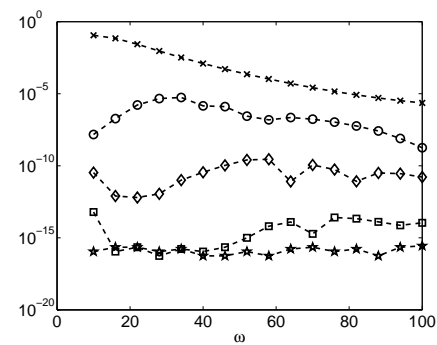

(e) $s=10$

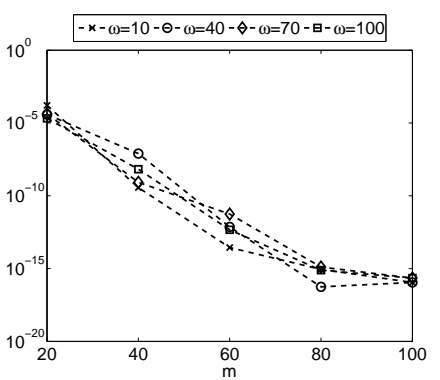

(b) $s=4$

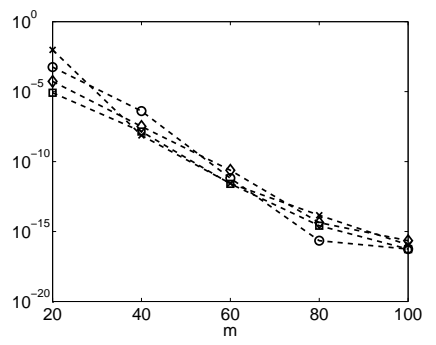

(d) $s=7$

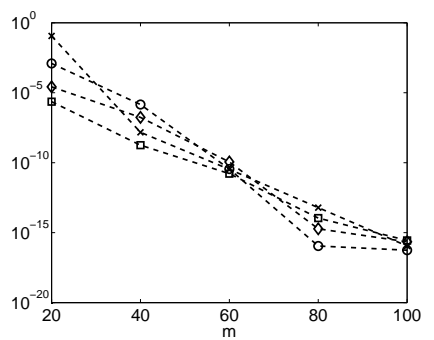

(f) $s=10$

Figure 4: Convergence of the superinterpolation scheme with Chebyshev points for the integral $I_{2}$ as a function of increasing $\omega$ (left figures) or as a function of increasing number of Chebyshev points $m$ (right figures). The errors behave asymptotically as $\mathcal{O}\left(\omega^{-3}\right), \mathcal{O}\left(\omega^{-5}\right)$ and $\mathcal{O}\left(\omega^{-7}\right)$ respectively for $s=4,7,10$.

[2] N. Bleistein and R. Handelsman. Asymptotic expansions of integrals. Holt, Rinehart and Winston, New York, 1975.

[3] S. N. Chandler-Wilde and D. C. Hothersall. Efficient calculation of the Green function for acoustic propagation above a homogeneous impedance plane. J. Sound Vibration, 180(5):705-724, 1995.

[4] R. Cools and A. Haegemans. Algorithm 824: CUBPACK: A package for automatic cubature; framework description. ACM Trans. Math. 
Softw., 29(3):287-296, 2003.

[5] K. T. R. Davies, M. R. Strayer, and G. D. White. Complex-plane methods for evaluating highly oscillatory integrals in nuclear physics. I. J. Phys. G: Nucl. Phys., 14(7):961-972, 1988.

[6] P. J. Davis and P. Rabinowitz. Methods of numerical integration. Computer Science and Applied Mathematics. Academic Press, New York, 1984 .

[7] A. Deaño and D. Huybrechs. Complex Gaussian quadrature of oscillatory integrals. Numer. Math., 112(2):197-219, 2009.

[8] A. Deaño, D. Huybrechs, and A. B. J. Kuijlaars. Riemann-Hilbert analysis of complex orthogonal polynomials associated with Gaussian quadrature. Technical Report TW-557, K.U.Leuven, January 2010.

[9] V. Domínguez, I. G. Graham, and V. P. Smyshlyaev. Stability and error estimates for Filon-Clenshaw-Curtis rules for highly-oscillatory integrals. Technical Report 02/10, Bath Institute for Complex Systems, 2010.

[10] J. D. Donaldson and D. Elliott. A unified approach to quadrature rules with asymptotic estimates of their remainders. SIAM J. Numer. Anal., 9:573-602, 1972.

[11] G. A. Evans and J. R. Webster. A comparison of some methods for the evaluation of highly oscillatory integrals. J. Comput. Appl. Math., 112(1):55-69, 1999.

[12] A. Gil, J. Segura, and N. M. Temme. Numerical methods for special functions. SIAM, Philadelphia, 2007.

[13] G. H. Golub and J. H. Welsch. Calculation of Gauss quadrature rules. Math. Comp., 23(106):221-230, 1969.

[14] D. Huybrechs and S. Olver. Highly oscillatory quadrature. In B. Engquist, A. Fokas, E. Hairer, and A. Iserles, editors, Highly Oscillatory Problems, pages 25-50, Cambridge, 2009. Cambridge Univ. Press.

[15] D. Huybrechs and S. Vandewalle. On the evaluation of highly oscillatory integrals by analytic continuation. SIAM J. Numer. Anal., 44(3):10261048, 2006.

[16] A. Iserles and S. P. Nørsett. On quadrature methods for highly oscillatory integrals and their implementation. BIT, 44(4):755-772, 2004.

[17] A. Iserles and S. P. Nørsett. Efficient quadrature of highly oscillatory integrals using derivatives. Proc. R. Soc. Lond. A, 461:1383-1399, 2005. 
[18] J. C. Mason and D. C. Handscomb. Chebyshev polynomials. Chapman and Hall/CRC, 2003.

[19] J. M. Melenk. On the convergence of filon quadrature. J. Comput. Appl. Math., pages 1-10, 2009. To appear.

[20] F. W. J. Olver. Asymptotics and special functions. Academic Press, Inc, New York, 1974.

[21] F. W. J. Olver, D. W. Lozier, R. F. Boisvert, and C. W. Clark. NIST Handbook of Mathematical Functions. Cambridge University Press, 2010.

[22] S. Olver. Moment-free numerical approximation of highly oscillatory integrals with stationary points. Euro. J. Appl. Maths, 18:435-447, 2006 .

[23] S. Olver. Fast, numerically stable computation of oscillatory integrals with stationary points. BIT, 50:149-171, 2010.

[24] A. Talbot. The accurate numerical inversion of Laplace transforms. J. Inst. Math. Appl., 23(1):97-120, 1979.

[25] R. Wong. Asymptotic approximation of integrals. SIAM, Philadelphia, 2001.

[26] S. Xiang. Efficient Filon-type methods for $\int_{a}^{b} f(x) \mathrm{e}^{\mathrm{i} \omega g(x)} \mathrm{d} x$. Numerische Mathematik, 105(4):658, 2007. 